\title{
Improved Detection Sensitivity in Functional MRI Data Using a Brain Parcelling Technique
}

\author{
Guillaume Flandin ${ }^{1,2,3}$, Ferath Kherif ${ }^{2,3}$, Xavier Pennec ${ }^{1}$, \\ Grégoire Malandain $^{1}$, Nicholas Ayache ${ }^{1}$, and Jean-Baptiste Poline ${ }^{2,3}$ \\ 1 INRIA, Epidaure Project, Sophia Antipolis, France \\ \{flandin, pennec, malandain, ayache\}@sophia.inria.fr \\ 2 Service Hospitalier Frédéric Joliot, CEA, Orsay, France \\ $\{$ kherif, poline\}@shfj.cea.fr \\ 3 IFR 49, Neuroimagerie fonctionnelle, Paris, France
}

\begin{abstract}
We present a comparison between a voxel based approach and a region based technique for detecting brain activation signals in sequences of functional Magnetic Resonance Images (fMRI). The region based approach uses an automatic parcellation of the brain that can incorporate anatomical constraints. A standard univariate voxel based detection method (Statistical Parametric Mapping [5]) is used and the results are compared to those obtained when performing detection of signals extracted from the parcels. Results on a fMRI experimental protocol are presented and show a greater sensitivity using the parcelling technique. This result remains true when the data are analyzed at several resolutions.
\end{abstract}

\section{Introduction}

Since the discovery of fMRI [10], the analysis of these series of images has been an extremely active field of research. One of the challenge when analyzing these noisy data is to detect very small increase of activity in the brain. Many approaches have been proposed for this purpose. They can be classified in several overlapping categories such as multivariate versus univariate, voxel based versus region based, parametric versus non parametric, inferential versus exploratory, etc.

Despite the number of approaches proposed, the only one that is extensively used to detect the brain activity is to fit at each and every voxel in the brain a linear model that represents the expected voxel time course derived from the experimental paradigm. A test is then applied to detect voxels that have a significant part of their variance explained by the model. An obvious problem with this technique is that the threshold controlling for the type one error (false positive rate) has to be adjusted depending on the number of tests performed, here as many as voxels in the brain (in the order of 20000). Because voxels are spatially dependent, techniques have been developed to set this threshold such that the correction for multiple comparison does not lead to a conservative test with reduced sensitivity. For this purpose, the theory of random field has been extensively developed and applied in particular through the work of K. Worsley [13]. 
This approach has been popularized by K. Friston and co-workers through the distribution of the SPM package1.

Because brain regions activated by a given experimental paradigm generally spread over many contiguous voxels, spatial regularization is used through the application of spatial filters. These filters tend to increase the signal to noise ratio (SNR) and permit a partial overlapping of the signal originating from different subjects data that are not (and cannot be) perfectly aligned [7. The correction for multiple comparison is strongly linked to the size of the spatial filter since the greater the dependence of the data, the less severe is the test correction. Clearly, activated region with size and shape similar to the one of the filter are best detected. Since activated regions can in principle have any size or shape, multi-filtering or multi-scale approaches have been investigated 1114. However, the greater the filter size the less precise are the boundaries of the region.

In this work, we propose an alternative approach that consists in parcelling the analyzed brain into a user defined number of regions (or parcels). The parcelling method is able to take into account anatomical information extracted from the $\mathrm{T}_{1}$-weighted MRI acquired together with the functional images. This should allow for the use of relatively large spatial averaging informed by the anatomy. It also should solve easily for the multiple comparison problem since functional signals extracted from parcels can in a first approximation be considered independent. In the first section, we present the parcelling technique based on a K-means algorithm with geodesic distances. The functional signal assignment to the parcels and the detection model are then presented, followed by a description of the methodology used to compare voxel and parcel based detection analyses. Comparison has been investigated with several filter sizes and corresponding numbers of parcels on an experimental protocol. We show that the method is more sensitive at several resolutions.

\section{Methods}

\subsection{Automatic Parcellation of Brain Images}

The aim is to provide a fully automatic parcellation of a certain volume of interest (the cortex in our case) at a (user supplied) adjustable resolution. Furthermore, a desired feature of such a parcellation is to obtain "homogeneous" parcels: a first intuitive guess is to use a criterion based on the cells volume but, to prevent the algorithm from finding very elongated cells, a criterion such as the sum of inertia of each cell (intra-class variance) seems more relevant, thus introducing a cell compactness concept. We propose an algorithm based on the K-means clustering [3] using geodesic distances.

Definition of the Volume of Interest. Here we restrict the functional data analysis to the cortex. A segmentation of the grey matter is performed on a $\mathrm{T}_{1}$ weighted image 9] and a brain mask is extracted from functional images using

\footnotetext{
${ }^{1}$ http://www.fil.ion.ucl.ac.uk/spm/
} 
an adapted threshold. The domain to parcel is then defined at an anatomical resolution as the intersection (logical and) between these two binary images.

Parcellation of the Domain. The volume of interest is described by a set of $3 \mathrm{D}$ coordinates $\left\{x_{i}\right\}$. We define parcels as connected clusters of anatomical voxels, represented by their centers of mass $\bar{x}_{j}$. The problem is then to find simultaneously a partition of the voxels $\left\{x_{i}\right\}$ into $k$ classes $C_{j}$ and the cell positions $\bar{x}_{j}$ minimizing the intra-class variance:

$$
I_{\text {intra }}=\sum_{j=1}^{k} \sum_{i \in C_{j}} d^{2}\left(x_{i}, \bar{x}_{j}\right)
$$

Such an optimization problem is efficiently solved using the well-known K-means algorithm in the classification context. After an initialization step that randomly selects $k$ distinct voxels in the volume of interest as the initial cell positions, the criterion is solved using an alternate minimization of $I_{\text {intra }}$ over:

1. The partition of the data (given cell positions): each voxel $x_{i}$ is assigned to the class $C_{j}$ that minimizes the distance to its position $\bar{x}_{j}$.

2. The cell positions (given a data partition): the position $\bar{x}_{j}$ is chosen to minimize the variance of the $x_{i}$ 's assigned to this class.

With spatial data, each step of the K-means algorithm can be rephrased in terms of discrete geometry.

Step 1 consists in the construction of a Voronoï diagram. Due to the nonconvexity of the domain, we cannot use Euclidean distances for the computation of $d$. Indeed two points on opposite sides of a sulcus are close to each other in Euclidean terms but may be geodesically far apart. Thus the geodesic 3D distance (the shortest path within the volume of interest) is the most suitable. We implemented 3D discrete Voronoï diagram with geodesic distances using region growing and hierarchical queues adapted from [2].

Step 2 consists in computing the "geodesic center of mass" of each cell. Its value may be obtained by a gradient descent on the intra-class variance but in practice, most of the cells are still convex and the standard center of mass is a good approximation. In our implementation, we use the Euclidean center of mass as far as it stays within the cell, and a gradient descent otherwise.

Finally, the K-means clustering algorithm consists in repeating these two estimations until convergence, reached when voxels assignments to the cells are the same at two consecutive steps. There are proofs of the convergence even if the domain is non-convex, but in our case we use approximations with discrete distances that prevent a statement on convergence. We however observed that this algorithm always converges in a reduced number of iterations (typically a few dozens). Although implemented in 3D, we present, for display purpose, in figure 1 an example of convergence of the algorithm on a $2 \mathrm{D}$ cortex slice of an hemisphere with 50 parcels. The algorithm can be further improved by introducing additional anatomical constraints through the use of weighted geodesic distances. 

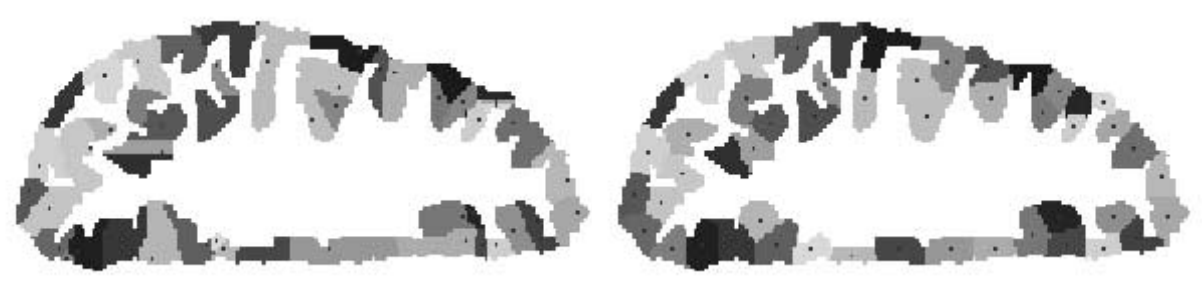

Fig. 1. 2D geodesic Voronoï diagram with 50 seeds (black dots). Left: random initialization. Right: after convergence of the K-means algorithm.

\subsection{Signal Assignment}

In this section, we address the method used to assign a representative time course at each parcel.

Parcellation being defined at an anatomical resolution, functional images are oversampled at this resolution using spline interpolation. Numerous strategies can then be used to provide a single temporal signal to each and every parcel using all signals belonging to the same cell: average them with mean or median, obtain the first eigenvector of their PCA or use multivariate analyses such as CCA (Canonical Correlation Analysis) to find the linear compound of signals that maximizes correlation with a model [4].

In the following, we used the sample mean in a first approximation leaving a thorough study of the best sampling technique for future work.

\subsection{Statistical Detection Model}

We first present the model used to detect activated signals coming from voxels or parcels. We used the General Linear Model (GLM) popularized by the SPM software. Then we present the multiple comparison problem corrections used for both voxel and parcel based analyses.

The General Linear Model. Given a column vector $Y$ corresponding to a time course, the general linear model [5] is:

$$
Y=X \beta+\epsilon
$$

where $X$ is the so-called design matrix whose columns represent the a priori information on expected signals, $\epsilon$ the residual error (assumed normally distributed) and $\beta$ the parameters of the model.

In order to take into account the temporal autocorrelation of functional time series, the data and the model are convolved by a Toeplitz smoothing matrix $K$ to impose a known autocorrelation $V=K K^{T}[6]$.

The maximum likelihood estimate of $\beta$, assuming a full rank design matrix, is:

$$
\hat{\beta}=\left(X^{* T} X^{*}\right)^{-1} X^{* T} K Y \quad \text { with } \quad X^{*}=K X
$$


A contrast $c$ of the parameter estimates is tested with:

$$
T=\frac{c^{T} \hat{\beta}}{\left(\operatorname{Var}\left[c^{T} \hat{\beta}\right]\right)^{1 / 2}}=\frac{c^{T} \hat{\beta}}{\left(c^{T} \hat{\sigma}^{2}\left(X^{* T} X^{*}\right)^{-1} X^{* T} V X^{*}\left(X^{* T} X^{*}\right)^{-1} c\right)^{1 / 2}}
$$

where $\hat{\sigma}^{2}$ is the estimated error variance. The effective degrees of freedom are given by $\nu=\frac{\operatorname{trace}(R V)^{2}}{\operatorname{trace}(R V R V)}$ with $R$ such as $\hat{\epsilon}=R Y$.

The null distribution of $\mathrm{T}$ may be approximated by a $t$-distribution with $\nu$ degrees of freedom. Thus hypotheses regarding contrasts of the parameter estimates can be tested and a threshold for a given voxel or parcel can be set to control the false detection rate.

Multiple Comparison Problem. Images contain a large number of voxels so that the risk of false positive across voxels will not be controlled if the statistical threshold is set as if only one test was performed: this is the so-called multiple comparison problem and is an important issue when comparing results provided by voxelwise (SPM) and parcelwise analyses. Multiple comparison correction depends on both the number of tests performed and their statistical dependence.

First, with a parcel based analysis, we may assume in a first approximation that signals are independent because, by construction, parcels signals are averaged over an important number of almost independent voxels. We can therefore apply a "Bonferroni" correction procedure that consists in adjusting the significance level $\alpha$ (type one error, usually $5 \%$ ) for the number of tests $n$ :

$$
\alpha_{\text {corrected }}=1-(1-\alpha)^{1 / n} \simeq \frac{\alpha}{n}
$$

Second, in a standard voxel-based analysis, the non independence of voxel intensities is obvious, due to both the initial resolution of images and to post processing smoothing. A Bonferroni correction would therefore be much too severe. A well-established correction proposed by Worsley [13] uses Random Field Theory to provide strong control over the type one error. An estimate of the field smoothness is computed, and the stringency of the correction depends on this measure and on the size of the volume analyzed. Smoothness based correction can be seen as the computation of the number of tests normalized for the global smoothness of the statistical field.

\subsection{Comparing Voxel and Parcel Based Techniques}

We investigated three different ways to perform such a comparison. Our goal is to provide a comparison of the detection with an equivalent spatial resolution. Given the very different natures of the two techniques, there are several ways of achieving such an equivalence. We briefly present three possible solutions:

- $\mathbf{C}_{1}$ : First, the number of parcels can be set such that it corresponds to the volume analyzed divided by a measure of the resolution of the filtered data 
used in the voxel based technique. The most natural measure in this instance is to consider the Full Width at Half Maximum of the point spread function (PSF) of the data. This corresponds to the applied filter combined with the intrinsic spatial dependency of the original images. When the former is large enough, the resulting number is close to the one imposed by the spatial filter. The number of parcels is set to the volume analyzed divided by the volume of the PSF at its half maximum.

- $\mathbf{C}_{2}$ : Second, the point spread function can be measured not on the original filtered volumes but on volumes corrected for the signal predicted by the model and normalized by their residual variance. This solution better reflects the statistical aspect of the problem. This corresponds to the notion of RESELS (Resolution Elements) in the work of K. Worsley 13. The number of parcels is set as the effective degrees of freedom [15].

- $\mathbf{C}_{3}$ : Lastly, we can use the (corrected) p-value threshold proposed by the random field theory, compute the corresponding number of independent tests $\left(N_{B}\right)$ and set the number of parcels to $N_{B}$.

We have investigated these three solutions and compared results obtained on the data described in the next section.

\subsection{Application: Task and Paradigm Design}

We compared detection results obtained in voxel and parcel based analyses on a cognitive paradigm that investigates the brain network involved in a motor (grasping) task [12. The experimental protocol consisted of three activation epochs separated by three control periods $(26 \mathrm{~s}$. each), preceded by a 4 -seconds instruction period. The subject performed this sequence twice. Repetition time (duration of one functional image acquisition) was 2 seconds for a total of 186 scans. Functional image matrix was $64 \times 64 \times 18$ with $3.75 \times 3.75 \times 3.8 \mathrm{~mm}^{3}$ voxel size. $\mathrm{A}_{1}$-weighted anatomical scan was acquired at the same time with a resolution of $0.94 \times 0.94 \times 1.5 \mathrm{~mm}^{3}(256 \times 256 \times 124$ matrix $)$.

Activations are investigated by setting a contrast between the control condition and the grasping one. The model $X$ used for detection consists in one function per condition (instruction, control, and task) modeled by a box-car regressor convolved with a canonical haemodynamic response function.

\section{Results and Discussion}

We applied several Gaussian filters (FWHM of 8, 12 and $16 \mathrm{~mm}$ ) on the raw functional images and computed the corresponding numbers of parcels for each comparison criterion $C_{1}, C_{2}$ and $C_{3}$. Results are similar for all filters and we therefore only present those corresponding to the $8 \mathrm{~mm}$ filtered data. Figure 2 shows results obtained with the SPM approach (top left) and the comparison with the parcel based approach with the 3 equivalent resolutions (1700 with criterion $C_{1}, 340$ with $C_{2}$ and 4900 with $C_{3}$ ).

We can observe a large increase in sensitivity for 340 and 1700 parcels. For instance, t-maps global maxima are respectively of 28.18 and 30.19 compared with 

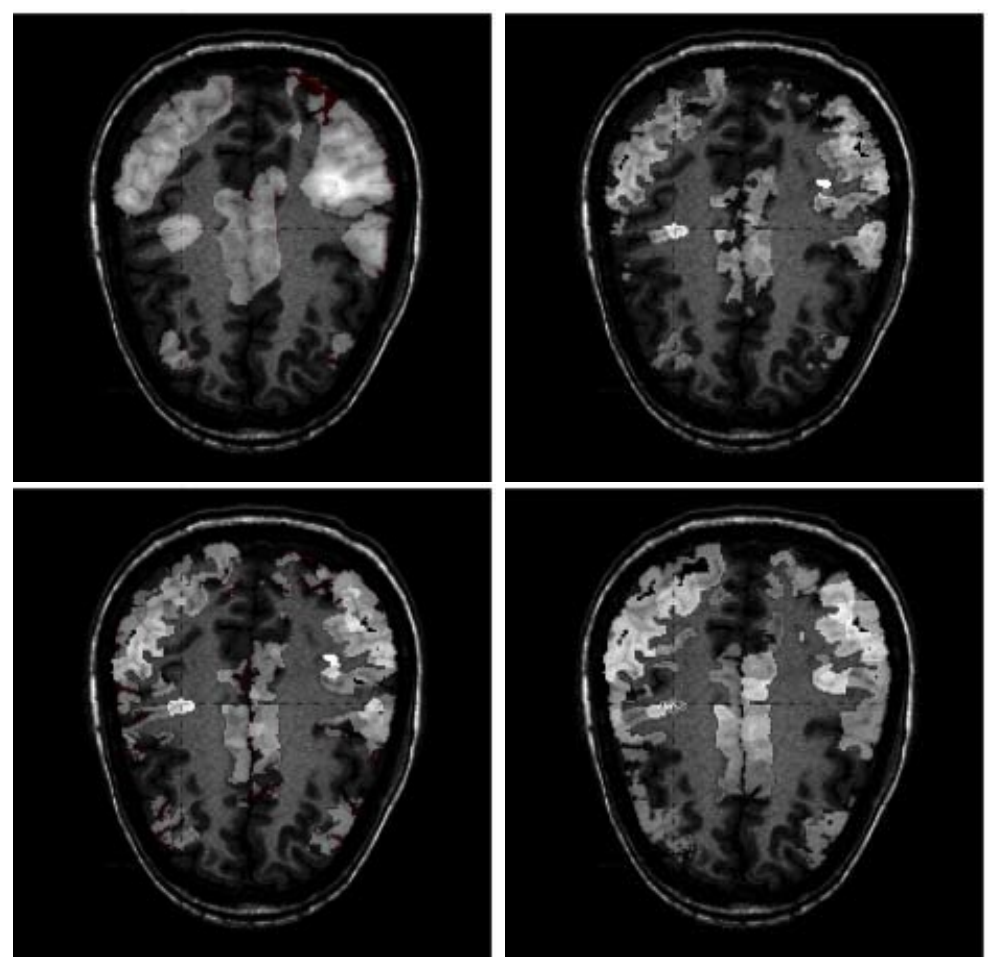

Fig. 2. Axial $T_{1}$-weighted MRI with detected activations superimposed $\left(p_{c}<0.05\right)$. SPM t-map with $8 \mathrm{~mm}$ smoothing (top left). Parcel-based t-map with 4900 parcels (top right), 1700 parcels (bottom left) and 340 parcels (bottom right).

23.25 using SPM, while the spatial localization does not seem to be degraded by the parcelling technique. With 4900 parcels, we obtain about the same sensitivity as SPM (global maxima is 22.5). However, in this case, the anatomical localization seems to be more precise with the parcel based approach. Indeed, 3D smoothing leads to the averaging of signals coming from different structures and to a poor localization of brain activity (look at the locus pointed by the cross in each image). We can maintain that the sensitivity increase is a consequence of the anatomically informed spatial smoothing performed by the parcelling technique (cortex structure taken into account), as opposed to 3D smoothing. Lastly, according to the results presented here, the criterion $C_{1}$ for the equivalent number of parcels (1700 parcels in this case) seems to be the most relevant.

\section{Conclusion}

This work is close in spirit with the methodology developed by Andrade [1] or Kiebel [8] performing a surface-oriented fMRI data analysis confined to the cortex since both techniques aim at incorporating anatomical information to the statistical analysis of functional time courses. The work [1] also reported 
increased sensitivity, but less pronounced than here. Furthermore we propose here a flexible method working at a voxel level that can be generalized to other structures than cortex, is more robust to misregistrations between functional and anatomical images and will eventually incorporate a priori information (anatomical and functional) in the parcellation definition.

\section{Acknowledgments}

The authors would like to thank J. Stoeckel for fruitful discussions. Many thanks also to O. Simon and S. Dehaene who provided the images.

\section{References}

1. A. Andrade, F. Kherif, J.-F. Mangin, K.J. Worsley, A.-L. Paradis, O. Simon, S. Dehaene, D. Le Bihan, and J.-B. Poline. Detection of fMRI activation using cortical surface mapping. Human Brain Mapping, 12:79-93, 2001.

2. O. Cuisenaire. Distance Transformations: Fast Algorithms and Applications to Medical Image Processing. PhD thesis, Katholieke Universiteit, Leuven, 1999.

3. R.O. Duda and P.E. Hart. Pattern Classification and Scene Analysis. Wiley, New York, 1973.

4. O. Friman, M. Borga, P. Lundberg, and H. Knutsson. Detection of neural activity in fMRI using maximum correlation modeling. NeuroImage, 15(2):386-395, 2002.

5. K.J. Friston, A.P. Holmes, J.-B. Poline, C.D. Frith, and R.S.J. Frackowiak. Statistical parametric maps in functional imaging: A general linear approach. Human Brain Mapping, 2:189-210, 1995.

6. K.J. Friston, O. Josephs, E. Zarahn, A.P. Holmes, S. Rouquette, and J.-B. Poline. To smooth or not to smooth? bias and efficiency in fMRI time-series analysis. NeuroImage, 12:196-208, 2000.

7. P. Hellier, C. Barillot, I. Corouge, B. Giraud, G. Le Goualher, L. Collins, A. Evans, G. Malandain, and N. Ayache. Retrospective evaluation of inter-subject brain registration. In MICCAI'01, volume 2208 of LNCS, pages 258-265, October 2001.

8. S.J. Kiebel, R. Goebel, and K.J. Friston. Anatomically informed basis functions. NeuroImage, 11(6):656-667, 2000.

9. J.-F. Mangin, V. Frouin, I. Bloch, J. Régis, and J. Lopez-Krahe. From 3D magnetic resonance images to structural representations of the cortex topography using topology preserving deformations. J. Math. Imaging and Vision, 5:297-318, 1995.

10. S. Ogawa, T.M. Lee, A.R. Kay, and D.W. Tank. Brain magnetic resonance imaging with contrast dependent on blood oxygenation. Proc Natl Acad Sci USA, 87:98689872, 1990.

11. J.-B. Poline and B.M. Mazoyer. Enhanced detection in brain activation maps using a multi filtering approach. J. Cereb. Blood Flow Metab., 14:639-641, 1994.

12. O. Simon, J.-F. Mangin, L. Cohen, D. Le Bihan, and S. Dehaene. Topographical layout of hand, eye, calculation, and language-related areas in the human parietal lobe. Neuron, 31(33(3)):475-87, Jan 2002.

13. K.J. Worsley, A.C. Evans, S. Marrett, and P. Neelin. A three-dimensional statistic analysis of CBF activation studies in human brain. J. Cereb. Blood Flow Metab., 12:900-918, 1992.

14. K.J. Worsley, S. Marrett, P. Neelin, and A.C. Evans. Searching scale space for activation in PET images. Human Brain Mapping, 4:74-90, 1996.

15. K.J. Worsley, J.-B. Poline, A.C. Vandal, and K.J. Friston. Tests for distributed, non-focal brain activations. NeuroImage, 2:183-194, 1995. 